\section{Kriminalität bei Jugendlichen: Nimmt die Gewalt zu?}

Beim Thema Jugendkriminalität gibt es viele unberechtigte Vorurteile. Auf dem Kongress der Deutschen Gesellschaft für Kinder- und Jugendmedizin stellten Experten aktuelle Daten vor.

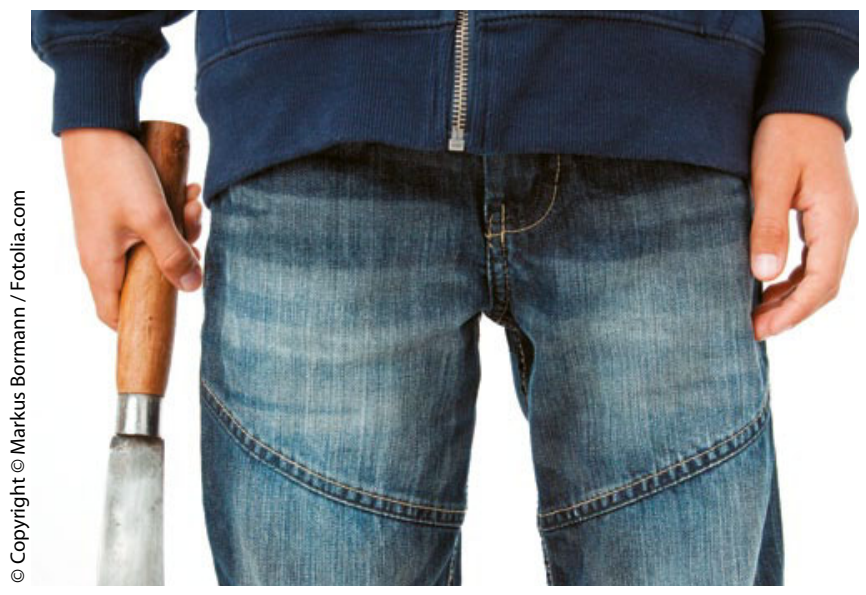

Die Zahl der jugendlichen Gewalttäter geht zurück.

Kriminalität unter Jugendlichen nimmt zu. Die jungen Straftäter werden gewalttätiger und brutaler, Mädchen holen gegenüber Jungen auf. Migranten sind dabei häufiger delinquent und schuld an der Entwicklung ist der gesteigerte (Gewalt-)Medienkonsum. Von diesen populären Gewissheiten gibt es eine ganze Reihe, erklärte Dirk Baier vom Kriminologischen Institut Niedersachsen (Hannover). Das Ergebnis seines Vortrags nahm er vorweg: „Diese Aussagen sind alle falsch."

Polizeistatistiken erfassen das so genannte Hellfeld, die Zahl der angezeigten Straftaten und damit nur etwa ein Viertel aller Fälle. Die allgemeine Anzeigebereitschaft hat sich in den vergangenen Jahren von 15 auf 20\% erhöht. Diese Tatsache kann eine Zunahme jugendlicher Gewalt suggerieren. Tatsächlich zeigen die Statistiken eine kontinuierliche und deutliche Abnahme beispielsweise der Rate von Ladendiebstählen. Gewalt und Sachbeschädigung - bis vor vier Jahren noch mit zunehmender Tendenz - sind seit 2007 ebenfalls rückläufig, so Baier: „Zum ersten Mal geht die Zahl registrierter jugendlicher Gewalttäter zurück."

Zum gleichen Ergebnis kam das Institut mit direkten Befragungen der Jugendlichen. Dabei wird auch das Dunkelfeld erfasst und das gesamte Ausmaß der Jugendkriminalität abgebildet: Die Kriminalität ist rückläufig, Gewalt (auch differenziert nach ethnischer
Zugehörigkeit) ist sowohl in ländlichen als auch in städtischen Gebieten gegenüber 1998 vermindert.

\section{Bildung als präventiver Faktor}

Die Ausnahme von der Regel bildet München: Hier ist die Zahl nicht-deutscher Gewalttäter, vor allem türkischer Abstammung, um mehr als $2 \%$ gestiegen. Eine Ursache dafür sah Dr. Nikolaus Weissenrieder aus München in den Schulverhältnissen der Stadt: „Wir haben es in den letzten acht Jahren im Vergleich zu anderen Städten nicht geschafft, das Bildungsniveau von Migranten zu verbessern. In München gibt es die höchste Zahl an Menschen mit Migrationshintergrund in den Hauptschulen."

In anderen Städten ist das Verhältnis gedreht worden. So hat sich etwa in Hannover die Zahl türkischstämmiger Abiturienten verdoppelt - bei gleichzeitiger Halbierung der Gewalttaten in dieser Gruppe. In München dagegen ist die entsprechende Zahl von Abiturienten um ein Drittel gesunken - bei Verdopplung der Gewalt. „Bildung als präventiver Faktor fällt hier aus", kommentierte Weissenrieder.

Für eine zunehmende Brutalisierung zeigt die Polizeistatistik keine Anhaltspunkte. Mord und Totschlag, ebenso wie Raub als brutale Gewalttaten kommen komplett zur Anzeige - sie sind konstant oder rückläufig. Bei sexueller Gewalt hat sich die Anzeigenquote in den letzten Jahren verdoppelt, die Dunkelfeldanalyse zeigt eine Abnahme der Fälle. Für sogenannte „Raufunfälle" auf dem Schulhof und daraus resultierende Frakturen belegen Daten der gesetzlichen Unfallversicherung einen deutlichen Rückgang, auch Körperverletzung ist seltener geworden. Das Geschlechterverhältnis bei Gewaltdelikten hat sich polizeistatistisch etwas angenähert und liegt heute bei 1:4,6 (Mädchen zu Jungen) gegenüber früher $1: 6$. Verantwortlich ist hier die erhöhte Bereitschaft, auch Mädchen anzuzeigen. Die Befragung von Jugendlichen ergibt dagegen, dass das Verhältnis konstant geblieben ist.

\section{Migrationsgruppen im Blick}

Die Kriminalstatistik unterscheidet lediglich zwischen deutschen und nicht-deutschen Jugendlichen; hier wird eine deutlich höhere Rate verschiedener Delikte bei nicht-deutschen Personen verzeichnet. Migranten werden aber auch wesentlich häufiger angezeigt, wie Baier erklärte. Im Dunkelfeld zeigen sich jedoch keine signifikanten Unterschiede außer bei den Gewalttäterraten.

Während Jugendliche asiatischer Herkunft die niedrigsten Raten aufweisen, ist die Quote bei türkischer und ehemals jugoslawischer/albanischer Abstammung hoch. Verantwortlich dafür sind die häufige Erfahrung elterlicher Gewalt, eine schlechtere sozialkulturelle Integration mit hoher Arbeitslosigkeit und niedrigem Bildungsniveau sowie Gewalt legitimierende Männlichkeitsnormen. Rechnet man diese Faktoren heraus, ergibt sich kein Unterschied zwischen den Gruppen. Faktoren wie Elterngewalt, Desintegration, delinquenter Freundeskreis und Männlichkeitsnormen, intensives Schulschwänzen, Drogen- und Alkoholkonsum sagen Gewalt deutlich besser voraus als der tatsächlich gestiegene Medienkonsum. Eine leichte Korrelation zwischen dem Konsum von Gewaltfilmen mit so genannten Ego-Shooter- oder Kampf-Spielen und Aggression gilt heute als belegt. Dieser Zusammenhang ist aber innerhalb eines multifaktoriellen Geschehens eher ein untergeordneter Faktor ohne direkten Einfluss auf das Gewaltverhalten, fasste Baier zusammen. „Auch Nicht-Spieler sind Täter, und umgekehrt sind nicht alle Vielspieler Täter." Michael Koczorek

Baier D, Weissenrieder N. Gewalt und Jugendliche 